\title{
Hernia de disco lumbar en un niño con fibrosis quística. Caso clínico
}

\author{
Lumbar disc herniation in a child with cystic fibrosis. Case report
}

Dr. George A. Alexiou ${ }^{a}$, Dra. Kalliopi Stefanaki ${ }^{b}$, Dr. George Sfakianos ${ }^{a}$ y Dr. Neofytos Prodromou ${ }^{a}$

\begin{abstract}
RESUMEN
Presentamos el caso de un niño con fibrosis quística y hernia de disco lumbar. Un niño de 8 años de edad consultó por dolor lumbar que aumentaba con la tos, al sentarse, caminar o inclinarse, y disminuía al acostarse. La prueba de levantamiento dela pierna en extensión fue positiva cuando el miembroinferior derechollegó a 60 grados. La prueba contralateral fue negativa. La resonancia magnética nuclear mostró una protrusión central del disco intervertebral entre L5-S1. El tratamiento conservador no fue efectivo, por lo cual se efectuó tratamiento quirúrgico, que hizo desaparecer el dolor. Según nuestro conocimiento, este es el primer caso comunicado de hernia de disco lumbar en un niño con fibrosis quística. Aunque este caso podría ser una coincidencia, se debe realizar una investigación detallada ante el dolor de espalda, síntoma frecuente en pacientes con fibrosis quística.

Palabras clave: hernia de disco lumbar, niños, fibrosis quística.
\end{abstract}

\begin{abstract}
We report a case of a child with cystic fibrosis and lumbar disc herniation. An 8-year-old boy presented with low back pain that exacerbated on coughing, sitting, walking, or bending and diminished when lying down. The straight leg raising test was positive when the right leg was lifted at 60 degrees. Crossed leg raising test was negative. Lumbar magnetic resonance imaging revealed a L5-S1 central disc protrusion. Conservative treatment was not effective and the patient underwent surgery. Postoperatively the patient experienced regression of the pain. To the best of our knowledge this is the first reported case of lumbar disc herniation in a child with cystic fibrosis. Although this case might be coincidental, thorough investigation of back pain, which is frequent in patients with cystic fibrosis, should be performed.
\end{abstract}

Key words: lumbar disc herniation, children, cystic fibrosis.

http:/ /dx.doi.org/10.5546/aap.2014.e43

a. Departamento de Neurocirugía.

b. Departamento de Patología.

Hospital de Niños "Agia Sofia”, Atenas, Grecia.

E-mail Address:

Dr. George Alexiou: alexiougrg@yahoo.gr.

Conflicto de intereses: ninguno que declarar.

Recibido: 30-7-2013

Aceptado: 15-10-2013

\section{INTRODUCCIÓN}

La hernia de disco lumbar es una entidad común en adultos, pero extremadamente infrecuente en niños. ${ }^{1}$ En ellos, las causas no son claras; se ha comunicado un retraso en el diagnóstico dado lo infrecuente de esta entidad en la edad pediátrica. ${ }^{1}$ La fibrosis quística es una enfermedad autosómica recesiva que afecta a $1 / 2500$ recién nacidos; los pulmones son uno de los órganos más afectados. El dolor de espalda es frecuente en pacientes con fibrosis quística. ${ }^{2}$ Recientemente, Denne et al. comunicaron sobre tres pacientes adultos con fibrosis quística que presentaron hernia de disco lumbar en el curso de su enfermedad. ${ }^{2}$ Presentamos el primer caso de hernia de disco lumbar tratado quirúrgicamente en un niño con fibrosis quística.

\section{CASO CLÍNICO}

Niño de 8 años de edad con diagnóstico de fibrosis quística desde los 3 años (estudio genético con identificación de la mutación F508), que consultó por dolor en la región lumbar y glútea, y deambulación anormal, que empeoraron gradualmente en los últimos 3 meses. Su peso corporal estaba en el percentilo 10-25 para su edad. El niño tenía tos persistente y había estado hospitalizado 2 meses atrás por infección respiratoria. El dolor aumentaba al sentarse, caminar o inclinarse y disminuía en posición horizontal, con las rodillas flexionadas. Además, se intensificaba con la tos. No refería trastornos de la función intestinal ni vesical, ni tenía fiebre. El examen físico mostró conservación de la fuerza y sensibilidad en los miembros. La prueba de levantamiento de la pierna en extensión fue positiva para la pierna derecha al llegar a los 60 grados. La prueba contralateral fue negativa. El reflejo tendinoso de Aquiles del lado derecho estaba disminuido. El diagnóstico diferencial incluyó traumatismo, estiramiento muscular, infección urinaria, síndrome viral, absceso espinal, hernia de disco y causas idiopáticas. 
El tratamiento conservador no fue efectivo para aliviar los síntomas. La resonancia magnética nuclear mostró una protrusión central del disco intervertebral entre L5-S1 con compresión del saco tecal (Figuras 1 y 2). La intervención quirúrgica consistió en una laminectomía a nivel de L5 y discectomía en L5-S1 para descomprimir el canal espinal. El examen histológico mostró fragmentos de cartílago degenerativo y tejido conectivo, con degeneración focal mixoide y microquística. Había también zonas con condrocitos de apariencia degenerativa y neovascularización. La tinción con azul alcián fue positiva en las áreas con patrón mixoide.

En el postoperatorio, desapareció el dolor. Durante el seguimiento hasta los 6 meses, no hubo signos de recurrencia.

\section{DISCUSIÓN}

El dolor de espalda es poco frecuente en niños y adolescentes sanos. ${ }^{3}$ Szpalski et al. estudiaron prospectivamente 287 niños de 9 a 12 años durante un período de dos años y encontraron que el 17,8\% tuvo al menos un episodio de dolor lumbar. ${ }^{3}$

FIgURA 1. Resonancia magnética nuclear con difusión, corte sagital de la columna lumbar en T2. Hernia del disco L5-S1 (flecha)

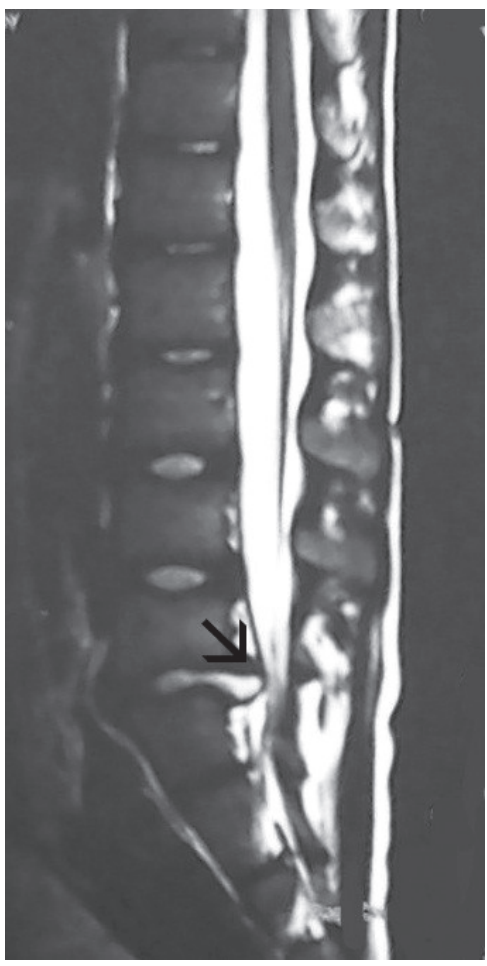

El diagnóstico diferencial incluye traumatismo, estiramiento muscular, crisis de anemia falciforme, causa idiopática, infección del tracto urinario, síndrome viral y enfermedad grave espinal, como tumores o infecciones. ${ }^{3,4}$ La historia clínica y el examen físico detallados pueden orientar el diagnóstico. La hernia de disco lumbar es extremadamente infrecuente en niños. En menores de 25 años, la frecuencia de hernias de disco lumbar que requieren cirugía es 5,5/100 000 personas-año. ${ }^{5}$

La causa más frecuente de hernia de disco lumbar en este grupo etario es el traumatismo, seguido por las malformaciones congénitas, la predisposición familiar y el deslizamiento de la epífisis vertebral. ${ }^{1}$ Los pacientes se presentan habitualmente con dolor lumbar, radiculopatía y pérdida motora y sensitiva. El examen de los reflejos tendinosos profundos puede mostrar disminución de los reflejos según la raíz comprometida. La prueba de estiramiento de la pierna en extensión es una de las más importantes para el diagnóstico de hernia de disco lumbar. Los estudios electromiográficos también son útiles en el diagnostico de radiculopatía lumbar dado que son muy específicos y constituyen un buen complemento de la resonancia magnética nuclear. ${ }^{6}$ Este es el examen de elección para el diagnóstico de hernia de disco lumbar.

El tratamiento conservador incluye el reposo en cama y la administración de antiinflamatorios

FIgURA 2. Resonancia magnética nuclear con difusión, corte axial en T2. Hernia del disco con marcado impacto en el canal neural (flecha)

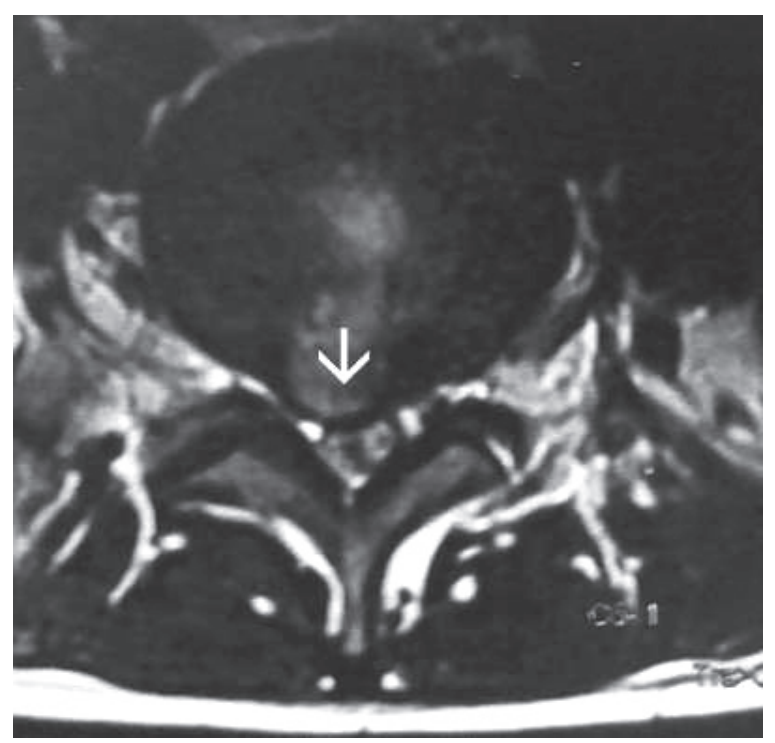


no esteroideos. La cirugía está indicada en caso de fracaso del tratamiento conservador durante 3 meses, dolor incontrolable y/o deterioro neurológico progresivo. ${ }^{1}$ Según la localización y el tamaño del disco prolapsado, se puede realizar una discectomía o microdiscectomía. ${ }^{7}$ Con respecto a la evolución, en un estudio prospectivo que compara niños y adultos, el grupo pediátrico tuvo un mejor posoperatorio, tanto desde el punto de vista clínico como radiológico. ${ }^{8}$

La fibrosis quística es una de las enfermedades hereditarias autosómicas recesivas más frecuentes entre los caucásicos. Con la mejoría de los índices de supervivencia, el dolor crónico se ha convertido en un problema común en los pacientes con esta enfermedad. ${ }^{9} \mathrm{El}$ dolor lumbar es una consulta habitual en pacientes con fibrosis quística. ${ }^{9,10}$ En los adultos, el dolor lumbar se atribuye generalmente a problemas de mineralización ósea, tos crónica y uso de esteroides. ${ }^{10}$ La hernia de disco lumbar en la fibrosis quística se ha comunicado con anterioridad solo en un estudio que incluyó a 3 pacientes adultos. ${ }^{2}$

Con respecto al efecto del dolor lumbar en pacientes con fibrosis quística, Denne et al. comunicaron el caso de un paciente con hernia de disco lumbar que murió por el deterioro de su situación pulmonar, dado que las técnicas de drenaje pulmonar no podían realizarse adecuadamente por el dolor. ${ }^{2}$

En niños con fibrosis quística, el dolor se comunicó en el 59\% de los pacientes; el dolor lumbar fue el principal componente en 16/73 $(22 \%)$ niños. ${ }^{11}$

De acuerdo con nuestro conocimiento, este es el primer caso comunicado de hernia de disco lumbar en un niño con fibrosis quística. Aunque podría ser una coincidencia, las tos crónica es considerada un factor de riesgo para la hernia de disco lumbar. ${ }^{12}$

Además, el disco intervertebral está compuesto por un núcleo pulposo altamente hidratado, rodeado por un anillo fibroso y una placa terminal cartilaginosa. ${ }^{13}$ Las células del núcleo pulposo son redondas y se encuentran en una matriz rica en proteoglicanos, mientras que las células del anillo fibroso son más alargadas y están anidadas en una matriz de fibras colágenas, y la placa terminal es una capa de cartílago. ${ }^{13}$ En la fibrosis quística, la arquitectura de la matriz extracelular puede ser anormal y puede estar afectada la sulfación de los glicoconjugados en algunos órganos y la utilización de los sulfatos en otros. ${ }^{14}$
Además, los pacientes con fibrosis quística tienen desmineralización ósea y desbalance entre la formación y degradación ósea. ${ }^{15}$ Por eso, mientras un disco intervertebral sano balancea el lento intercambio entre síntesis y degradación de la matriz, en la fibrosis quística puede existir un desbalance en este aspecto.

En conclusión, el dolor lumbar debe ser cuidadosamente estudiado en la edad pediátrica y la hernia de disco lumbar debe investigarse aunque sea infrecuente.

En la fibrosis quística, el dolor lumbar es un síntoma común, pero debe ser cuidadosamente estudiado.

\section{BIBLIOGRAFÍA}

1. Haidar R, Ghanem I, Saad S, Uthman I. Lumbar disc herniation in young children. Acta Paediatr 2010;99:19-23.

2. Denne C, Vogl-Voswinckel AE, Wurmser H, Steinborn M, et al. Lumbar disc herniation in three patients with cystic fibrosis: a case series. J Med Case Rep 2011;5:440.

3. Szpalski M, Gunzburg R, Balague F, Nordin M, et al. A 2-year prospective longitudinal study on low back pain in primary school children. Eur Spine J 2002;11:459-464.

4. Selbst SM, Lavelle JM, Soyupak SK, Markowitz RI. Back pain in children who present to the emergency department. Clin Pediatr (Phila) 1999;38:401-406.

5. Bruske-Hohlfeld I, Merritt JL, Onofrio BM, Stonnington $\mathrm{HH}$, et al. Incidence of lumbar disc surgery. A populationbased study in Olmsted County, Minnesota, 1950-79. Spine (Phila Pa 1976) 1990;15:31-35.

6. Barr K. Electrodiagnosis of lumbar radiculopathy. Phys Med Rehabil Clin N Am 2013;24:79-91.

7. Thomas JG, Hwang SW, Whitehead WE, Curry DJ, et al. Minimally invasive lumbar microdiscectomy in pediatric patients: a series of 6 patients. JNeurosurg Pediatr 2011;7:616-619.

8. Çelik S, Göksu K, Çelik SE, Emir CB. Benign neurological recovery with low recurrence and low peridural fibrosis rate in pediatric disc herniations after lumbar microdiscectomy. Pediatr Neurosurg 2011;47:417-422.

9. Ravilly S, Robinson W, Suresh S, Wohl ME, et al. Chronic pain in cystic fibrosis. Pediatrics 1996;98:741-747.

10. Tattersall R, Walshaw MJ. Posture and cystic fibrosis. J R Soc Med 2003;43:18-22.

11. Sermet-Gaudelus I, De Villartay P, de Dreuzy P, Clairicia M, et al. Pain in children and adults with cystic fibrosis: a comparative study. J Pain Symptom Manage 2009;38:281-90.

12. Heliövaara $M$, Knekt $P$, Aromaa A. Incidence and risk factors of herniated lumbar intervertebral disc or sciatica leading to hospitalization. J Chronic Dis 1987;40:251-8.

13. Pattappa G, Li Z, Peroglio M, Wismer N, Alini M, Grad S. Diversity of intervertebral disc cells: phenotype and function. J Anat 2012;221:480-96.

14. Hill WG, Harper GS, Rozaklis T, Boucher RC, et al. Organspecific over-sulfation of glycosaminoglycans and altered extracellular matrix in a mouse model of cystic fibrosis. Biochem Mol Med 1997;62:113-22.

15. Baroncelli GI, De Luca F, Magazzú G, Arrigo T, et al. Bone demineralization in cystic fibrosis: evidence of imbalance between bone formation and degradation. Pediatr Res 1997;41:397-403. 